\title{
A Preoperative Marking Template for Deep Inferior Epigastric Artery Perforator Flap Perforators in Breast Reconstruction
}

\author{
Benjamin H Miranda, Matthew Pywell, David Floyd \\ Royal Free London NHS Foundation Trust, London, UK
}

\begin{abstract}
Preoperative perforator marking for deep inferior epigastric artery perforator flaps is vital to the success of the procedure in breast reconstruction. Advances in imaging have facilitated accurate identification and preselection of potentially useful perforators. However, the reported imaging accuracy may be lost when preoperatively marking the patient, due to 'mapping errors', as this relies on the use of 2 reported vectors from a landmark such as the umbilicus. Observation errors have been encountered where inaccurate perforator vector measurements have been reported in relation to the umbilicus. Transcription errors have been noted where confusing and wordy reports have been typed or where incorrect units have been given (millimetres vs. centimetres). Interpretation errors have also occurred when using the report for preoperative marking. Furthermore, the marking process may be unnecessarily time-consuming. We describe a bespoke template, created using an individual computed tomography angiography image, that increases the efficiency and accuracy of preoperative marking. The template is created to scale, is individually tailored to the patient, and is particularly useful in cases where multiple potential suitable perforators exist.
\end{abstract}

Keywords Free tissue flaps / Perforator flaps / Mastectomy / Breast neoplasms / Tomography, X-ray computed

\author{
Correspondence: \\ Benjamin H Miranda \\ Royal Free London NHS Foundation \\ Trust, Royal Free Hospital, Pond \\ Street, London NW3 20G, UK \\ Tel: +44-(0)7961-996-229 \\ Fax: +44-(0)20-7830-2468 \\ E-mail: DrBMiranda@googlemail.com
}

No potential conflict of interest relevant to this article was reported.

Received: 21 Nov 2013 • Revised: 10 Dec 2013 • Accepted: 18 Dec 2013

pISSN: 2234-6163 • elSSN: 2234-6171 • http://dx.doi.org/10.5999/aps.2014.41.2.171 • Arch Plast Surg 2014;41:171-173

\section{INTRODUCTION}

The deep inferior epigastric artery perforator (DIEP) flap is the gold standard for breast reconstruction [1]. The selection of a suitable muscle perforator from the deep inferior epigastric artery is essential to flap viability. Historically, perforators were located preoperatively by Doppler, and directly at the time of dissection based on their calibre [1]. Advances in imaging, particularly computed tomography angiography (CTA), has facilitated preoperative perforator identification, allowing for improved flap design and shorter operating times [2]. At present, this is achieved by measuring the reported distances, using 2 vectors, inferior and lateral to the umbilicus, and marking these on the patient's abdomen.

Our unit has routinely used preoperative CTA for over 10 years. During this time, we have noted that this process may be unnecessarily time-consuming and that 'mapping errors' may occur to further decrease the efficiency of the preoperative perforator marking process. Observation errors have been encountered where inaccurate perforator vector measurements have been reported in relation to the umbilicus. Transcription errors have been noted where confusing and wordy reports have been

Copyright $($ C 2014 The Korean Society of Plastic and Reconstructive Surgeons

This is an Open Access article distributed under the terms of the Creative Commons Attribution Non-Commercial License (http://creativecommons.org/

licenses/by-nc/3.0/) which permits unrestricted non-commercial use, distribution, and reproduction in any medium, provided the original work is properly cited.

www.e-aps.org 
typed or where incorrect units have been given (millimetres vs. centimetres). Interpretation errors have also occurred when using the report for preoperative marking. The use of templates has been shown to be a simple, efficient and cost-effective way of increasing preoperative marking accuracy, reducing theatre time and improving outcomes, having previously been used for reconstruction of the infra-mammary fold and breast mound planning with DIEP flap reconstructions $[3,4]$.

We describe the use of a novel bespoke template, generated on a grid and taken in a standardised manner directly from CTA images, which increases the speed and accuracy of preoperative patient marking by overcoming DIEP perforator 'mapping errors.'

\section{IDEA}

Preoperative CTA is undertaken, with the umbilicus identifiable due to the use of contrast. After exclusion of the smallest perforators, the remaining suitable perforators are mounted onto a coronal-axis grid at umbilical level and reported by the radiology department (Fig. 1). After careful surgical review and perforator selection at a subcutaneous plane just anterior to the rectus sheath, the image is inserted, to scale, into a word processor template page with standardised margins and a built-in ruler

Fig. 1. Coronal computed tomography angiography, generated by radiology, with marked midline and umbilical axes (green lines) and perforators (blue arrows) (scale $=5 \mathrm{~cm}, 1$ $\mathrm{cm}^{2}$ grid)

There were 2 perforators on the left and 2 on the right hemi-abdomen, all perforating the rectus sheath: a $1.0-\mathrm{mm}$ calibre subcutaneous segment perforator $31 \mathrm{~mm}$ lateral and $18 \mathrm{~mm}$ inferior to the umbilicus on the right side (blue arrow), a 0.7-mm subcutaneous segment perforator $53 \mathrm{~mm}$ lateral and $19 \mathrm{~mm}$ inferior to the centre of the umbilicus on the right side (not marked), a 1.0- $\mathrm{mm}$ subcutaneous segment perforator $38 \mathrm{~mm}$ lateral and $45 \mathrm{~mm}$ inferior to the centre of the umbilicus on the left (blue arrow) and a 1.1- $\mathrm{mm}$ subcutaneous segment perforator $53 \mathrm{~mm}$ lateral and $26 \mathrm{~mm}$ inferior to the centre of the umbilicus (blue arrow).

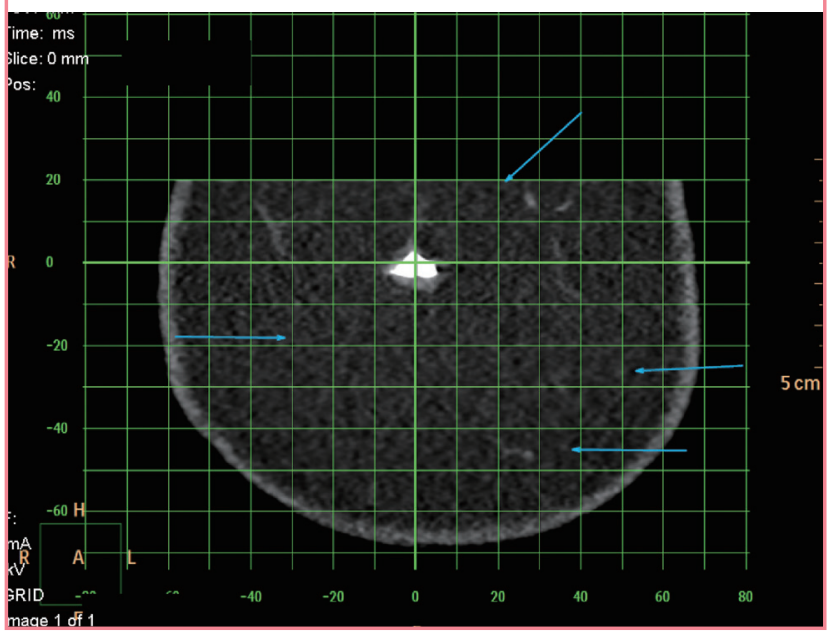

to ensure a 1:1 printing size without requiring size adjustment. The image is printed onto a transparent acetate sheet and holes 'punched' through the carefully selected perforators using a 3.0-mm biopsy punch (Stiefel Laboratories Inc., North Carolina, USA) (Fig. 2). The acetate is laid onto the abdomen, also marked preoperatively from the sternal notch to pubic symphysis, carefully centred at the umbilicus and lined up with the pa-

Fig. 2. Acetate print reproduction of generated computed tomography angiography image (to scale)

The 3 largest perforators, which were marked radiologically (arrows), have been cut out of the acetate by using a 3.0-mm biopsy punch (Stiefel Laboratories Inc., North Carolina, USA).

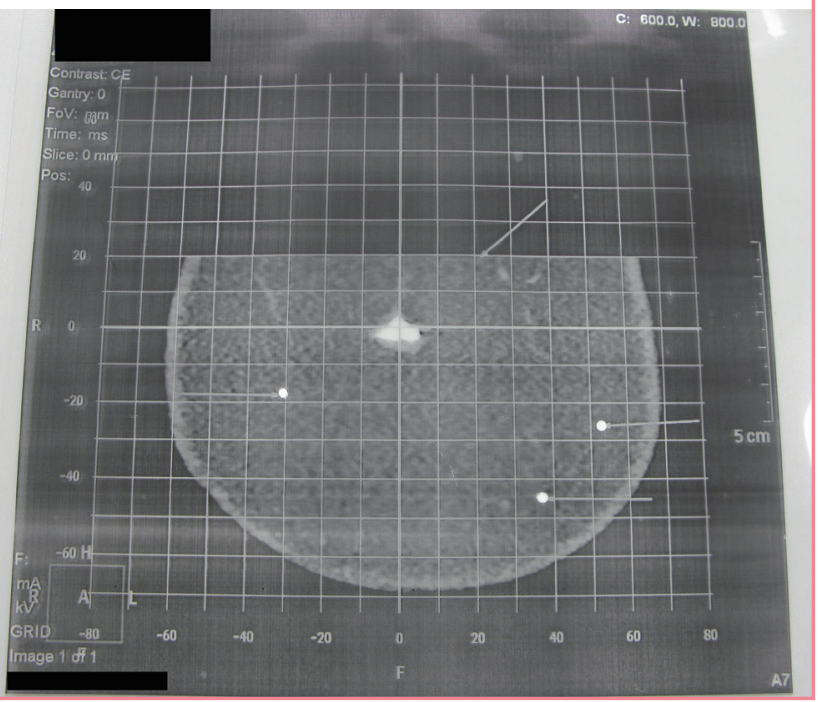

Fig. 3. Preoperative marking process

The 'punched' acetate is laid onto the abdomen, also marked preoperatively from sternal notch to pubic symphysis, carefully centred at the umbilicus and lined up with the patient's midline. The perforator sites are marked directly onto the abdomen through the punched holes.

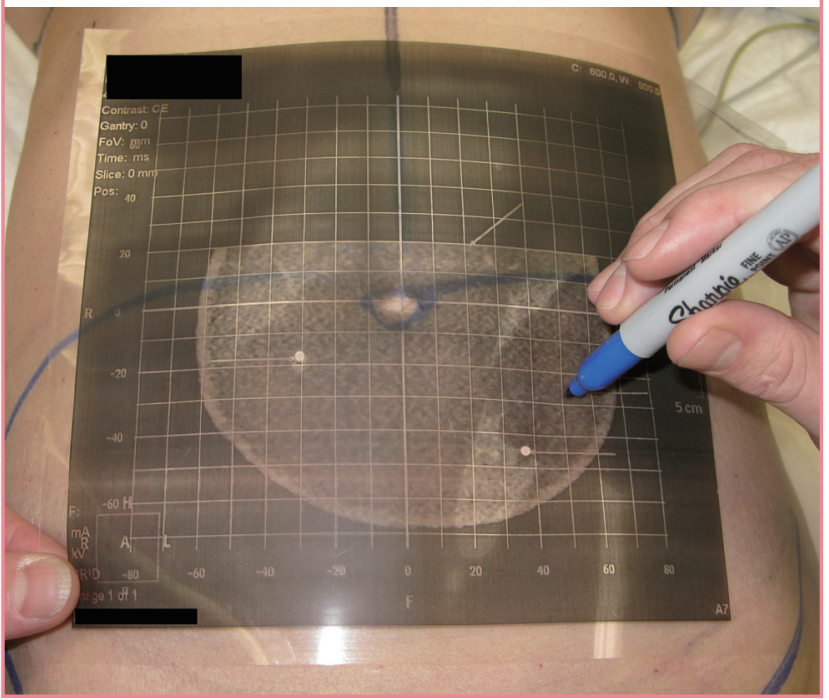




\section{Fig. 4. Completed preoperative marking}

After acetate removal, the most suitable perforator is quickly selected using a hand-held Doppler in preparation for surgery.

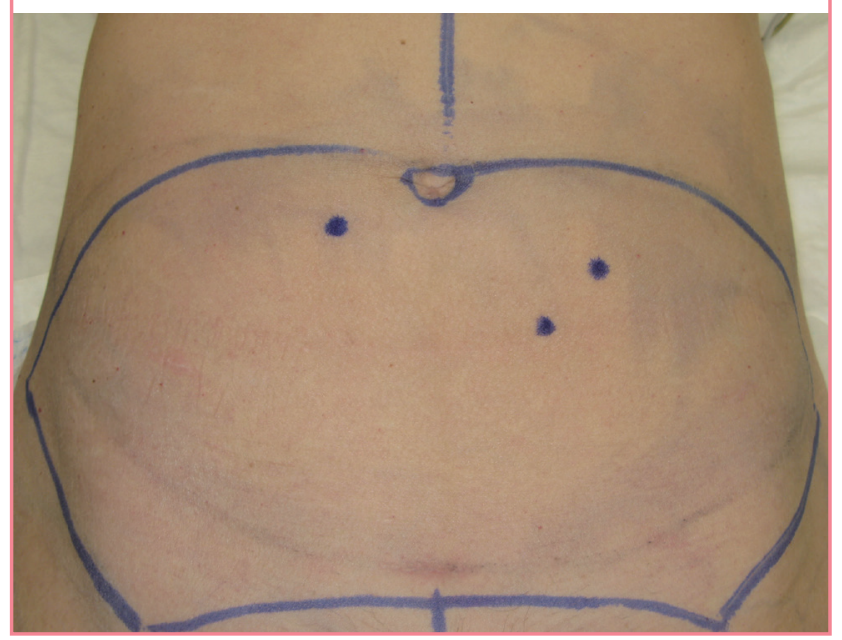

tient's midline. The perforator sites are marked directly onto the abdomen through the punched acetate holes (Figs. 3, 4). In all cases, perforator locations were confirmed with Doppler USS and found intraoperatively under the abdominal skin markings.

\section{DISCUSSION}

Preoperative DIEP perforator marking is currently undertaken using CTA reports of 2 vector distances from the umbilicus. These distances are measured directly onto the patient. This marking process may be time-consuming, particularly when multiple perforators exist. Furthermore, 'mapping errors' may occur due to errors in observation, transcription and interpretation. The use of templates in breast reconstruction has been shown to be a simple, efficient and cost-effective way to increase preoperative marking accuracy, reduce theatre time and improve outcomes, having previously been used for the reconstruction of the infra-mammary fold and breast mound planning with DIEP flap reconstructions $[3,4]$.

We have found this template technique to be easy to use and reproducible; further, it improves the accuracy and efficiency of preoperative marking for patients undergoing DIEP flap breast reconstruction.

\section{REFERENCES}

1. Koshima I, Soeda S. Inferior epigastric artery skin flaps without rectus abdominis muscle. BrJ Plast Surg 1989;42:645-8.

2. Hamdi M, Van Landuyt K, Van Hedent E, et al. Advances in autogenous breast reconstruction: the role of preoperative perforator mapping. Ann Plast Surg 2007;58:18-26.

3. Akhavani M, Sadri A, Ovens L, et al. The use of a template to accurately position the inframammary fold in breast reconstruction. J Plast Reconstr Aesthet Surg 2011;64:e259-61.

4. Tregaskiss A, Vermaak PV, Boulton R, et al. The template technique for breast mound planning when using abdominal flaps for breast reconstruction. Breast 2012;21:686-9. 